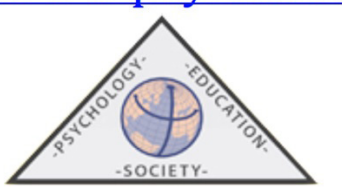

\title{
Estrategias para la reducción del estigma hacia la salud mental en el contexto escolar: estudio preliminar y pilotaje del programa "lo hablamos"
}

\author{
Jesús DE LA HIGUERA ROMERO, Andrea JiMÉNEZ GONZÁLEZ, \\ María ZURITA CARRASCO, Andrea CANDELAS MUÑOZ, Paula FUICA PEREG, \\ Eloísa MARTÍNEZ FERNANDEZ-REPETO, Salvador MORENO ALBERT, \\ Carmen H. ORTEGA HERREROS y Antonio LINARES COBACHO
}

\author{
Hospital Universitario de Puerto Real
}

(Recibido el 4 de Mayo de 2020, Aceptado el 20 de Julio de 2020)

\begin{abstract}
RESUMEN: Existe un creciente interés por las intervenciones educativas encaminadas a la reducción del estigma hacia los trastornos mentales en el contexto escolar. A pesar de la importante disparidad en cuanto a formatos y metodología de actuación, por lo general, este tipo de programas han demostrado ser una herramienta útil y costo-eficiente para favorecer la promoción de la salud mental del adolescente y que este reduzca sus prejuicios y miedos hacia la enfermedad. El presente trabajo describe el pilotaje del programa Lo Hablamos, una estrategia de intervención manualizada, con alto nivel de estructuración en sus contenidos y diseñada para la reducción del estigma hacia la enfermedad mental en las aulas. Participaron en el estudio un total de 315 alumnos de $3^{\circ}$ y $4^{\circ}$ de Enseñanza Secundaria Obligatoria, con edades comprendidas entre los 14 y 17 años. El programa se desarrolló en una única sesión de 3 horas dentro del horario lectivo habitual. Al finalizarla los participantes cumplimentaron una encuesta anónima diseñada para extraer sus valoraciones y preferencias sobre las actividades realizadas. Los resultados indican que la intervención es valorada de manera positiva por el alumnado, exhibiendo además las tareas y ejercicios que en ella se proponen un elevado nivel de comprensión y de dinamismo percibido. El programa parece tener una población diana situada entre los 14-16 años, por encima de estas edades se evidencia un mayor porcentaje de opiniones negativas. Los datos apuntan a que se trata de una herramienta interesante para la actuación sobre el estigma en el ámbito escolar.
\end{abstract}

Palabras clave: enfermedad mental; estigma; escuela.

\section{Strategies for Reducing Stigma To Mental Illness in a School Setting: Preliminary Study and Pilot of the "Lo Hablamos" Program}

ABSTRACT: There is a growing interest in reducing stigma towards mental disorders in the school context using educational interventions. Despite the significant variety of formats and methodology of action, in general, these types of programs have proven to be an useful and cost-efficient tool for 
promoting adolescent mental health, reducing their prejudices and fears towards the disease. The present work describes the pilot of the Lo Hablamos program, a manualized intervention strategy with a highlevel structure of content designed to reduce the stigma of mental illness in the classroom. A total of 315 students from the 3rd and 4th year of Obligatory Secondary Education, aged between 14 and 17, participated in the study. The program was developed in a single 3-hour session within regular school hours. At the end of the session, the participants completed an anonymous survey designed to extract their assessments and preferences about the activities carried out. The results indicate that students value the intervention positively, also the tasks and exercises shows a high level of understanding and perceived dynamism. The program seems to have a target population located between 14-16 years old, above these ages a higher percentage of negative opinions is evidenced. The data suggest that Lo Hablamos program is an interesting tool for action on stigma in the school environment.

Keywords: Mental Illness; Stigma; School.

Correspondencia: Andrea Jiménez González. Unidad de Gestión Clínica de Salud Mental. Calle Romería, 7, 11510. Cádiz. E-mail: andrea.jimenez.gonzalez.ssppa@juntadeandalucia.es

\section{Introducción}

A pesar de los importantes avances en la terapéutica y que las nuevas conceptualizaciones en salud mental, con el modelo de recuperación como base, están reivindicando cada vez con más fuerza el papel activo y esperanzado que pueden tener las personas afectadas, el peso de las antiguas visiones y muchos de los mitos tradicionalmente asociados a los problemas de salud mental siguen estando presentes entre la población general (Angermeyer et al., 2016; Baba et al., 2017; Barry, McGinty, Pescosolido y Goldman, 2014; Pescosolido, Manago y Monahan, 2019).

El sostenimiento de estas creencias estigmatizantes, los prejuicios y conductas discriminatorias a ellas asociados, puede terminar por provocar una conceptualización del otro como alguien diferente, incentivando el deseo de marcar distancia social y la necesidad de implementar medidas coercitivas o de protección. (Choudhry, Mani, Ming, y Khan, 2016; Corrigan, Bink, Fokuo y Schmidt, 2015; Schlier, y Lincoln, 2019; Walsh y Foster 2020).

La reducción del estigma se ha configurado como un objetivo relevante dentro de las políticas sanitarias de salud mental y se han planteado intervenciones específicas para actuar sobre el mismo. De manera general encontramos tres tipos de programas: por un lado, los que están diseñados como estrategias globales con varios contextos de actuación y mayor alcance poblacional como por ejemplo "Beyonblue" en Australia; (Corrigan, Powell y Al-Khouja, 2015),"Time to Change" en Reino Unido (González-Sanguino, Potts, Milenova, y Henderson, 2019; Sampogna et al., 2017) o "Opening Minds" en Canadá (Stuart et al., 2014; Szeto, Dobson, Luong, Krupa y Kirsh, 2019); por otro, los destinados a grupos específicos (adolescentes, estudiantes universitarios, profesionales de la salud, empleadores etc.) (Deb et al., 2019; Harris et al., 2019; Heim et al., 2019; Sugumar, Fleming y Ogden, 2019) y finalmente los programas individualizados para disminuir el autoestigma (Setti et al., 2019; Yanos, Lucksted, Drapalski, Roe y Lysaker, 2015). La propia naturaleza evolutiva del momento en el que se desarrollan hace que, a nuestro juicio, los programas destinados a la actuación en el ámbito escolar tengan una especial trascendencia e interés. La adolescencia es un periodo de cambio en el que comienzan a cristalizar las visiones de uno mismo y de los demás que posteriormente configurarán el estilo de vida adulto. Hoy día 
sabemos que el estigma hacia los problemas mentales no emerge de manera impredecible después de esta etapa, sino que las creencias, que tienen influencia en el desarrollo del proceso de estigmatización aparecen en estas edades para convertirse en estándares evaluativos con posterioridad (Bulanda, Bruhn, Byro-Johnson, y Zentmyer, 2014; Chandra y Minkowitz 2006; Georgakakou-Koutsonikou, y Williams, 2017; O’Driscoll, Heary, Hennessy y McKeage, 2012; Silke, Swords y Heary, 2016; Wahl, 2002). La presencia de estas actitudes no solo es relevante de cara al estereotipo adulto, sino que lo es también, en buena medida, porque si se activan en el medio escolar como respuesta a la diferencia percibida en el otro, van a provocar el rechazo potencial entre iguales dificultando aún más la adaptación de los jóvenes con problemas emocionales. La exclusión de las actividades de grupo, el rechazo o la evitación manifiesta pueden emerger como estilo de relación y actuar a la manera de un acoso escolar indirecto de especial relevancia, en una edad en la que la personalidad está en formación y se es especialmente vulnerable al estigma (Clement et al., 2015; Lansu, 2018; O’Driscoll, Heary, Hennessy, McKeague, 2015b). Las investigaciones que han intentado perfilar qué factores influyen en la construcción de estos estereotipos han puesto de manifiesto que el incremento de la edad tiene un efecto negativo sobre las actitudes y la distancia social expresada hacia el trastorno mental, existiendo además variabilidad en función de la tipología de problemas emocionales exhibidos (Dolphin y Hennessy, 2017; McKeague, Hennessy, O’Driscoll, y Heary, 2015). En la misma línea se ha constatado que, a diferencia de los varones, las chicas suelen ser más receptivas y muestran menos actitudes negativas hacia sus iguales con problemas emocionales (Kennedy y Belgamwar, 2014; O'Driscoll et al., 2012; Silke, Swords y Heary, 2017; Swords, Heary y Hennessy, 2011).

Son numerosas las experiencias que se vienen desarrollando para disminuir el estigma y promover la salud mental en el contexto escolar. A pesar de la enorme disparidad existente entre las mismas en cuanto a metodología, grado de estructuración, extensión y otros parámetros relevantes, en líneas generales en la mayoría de los trabajos se hace notar el efecto positivo inicial que tienen sobre las actitudes estigmatizantes, subrayándose la rentabilidad de las mismas (Andersen, 2013; Bentham, Daunt, Taylor y Simmons, 2013; Bulanda et al., 2014; Giannakopoulos et al., 2012; Gronholm, Nye, y Michelson, 2018; Hayes et al., 2019; Ke et al., 2015; Ojio et al., 2015; Puolakka, Haapasalo-Pesu, Konu, Åstedt-Kurki y Paavilainen, 2014; Sakellari, Sourander, Kalokerinou-Anagnostopoulou y Leino-Kilpi, 2014; Skre et al., 2013; Stuart et al., 2014; Yang, Cervera, Tye, Ekker y Pierret, 2018). En nuestro contexto se han publicado estudios de eficacia con programas como el "What's up" (Andrés-Rodríguez et al., 2017), el "EspaiJove" (Casañas et al., 2018) o el "Stigma-Stop" (Cangas et al., 2019; Cangas et al., 2017), incluyendo en algunos casos tecnologías como la realidad virtual como en el "Inclúyete VR" (Cangas y Galván, 2020). A pesar de todo, las revisiones sistemáticas y estudios de metaanálisis realizados hasta la fecha plantean un escenario de resultados más matizado, apuntando algunos elementos interesantes de cara a la optimización de las intervenciones (Corrigan et al., 2015; Corrigan y Lundin,2012; Giannakopoulos et al., 2012; Griffiths, Carron-Arthur, Parsons y Reid, 2014; Hanisch et al., 2016; Schiffman, Stephan, Hong y Reeves, 2015; Hartog, Hubbard, Krouwer, Thornicroff, Kohrt y Jordans, 2020; Mehta et al., 2015; Mellor, 2014; Morgan, Reavley, Ross, San Too y Jorm, 2018; Salerno, 2016; Schachter, 2008). Se suelen reseñar varias limitaciones: la primera de ellas tiene que ver con la pobre calidad metodológica de buena parte de los estudios realizados hasta la fecha, apuntándose la necesidad de manualizar las intervenciones y someterlas a estudios ran- 
domizados para evaluar su eficacia. La segunda se refiere a que pocos estudios aportan seguimiento de resultados y cuando lo hacen se muestra, de manera consistente, un descenso en la eficacia de los mismos, algo que hace pensar sobre la necesidad de estructurar actuaciones más extensivas. La última limitación alude a los instrumentos utilizados para la evaluación del cambio, prácticamente en todos los casos medidas de auto informe, que lo que reflejan en realidad son las intenciones conductuales y están muy sujetas a efectos de deseabilidad social en las respuestas, planteándose la necesidad de incorporar medidas más objetivas de cambio conductual. Para optimizar las intervenciones, se señala como necesario superar lo que algunos autores denominan el "modelo de buenas intenciones" (Corrigan et al., 2016) y comenzar a estructurar los programas sobre la base del conocimiento científico acumulado, tanto en lo referente a su estructura como a sus contenidos. En esta línea se han ido perfilando algunos de los factores que parecen tener más peso en la varianza resultados (Knaak, Modgill y Patten, 2014; Mittal, Corrigan, Drummond, Porchia y Sullivan, 2016; Pinfold, Thornicroft, Huxley y Farmer, 2005; Ross, Hart, Jorm, Kelly, y Kitchener, 2012; Thornicroft et al., 2019; Thornicroft et al., 2016; Weare y Marklam, 2005; Woolfson, Woolfson, Mooney y Bryce, 2009), entre ellos destacan el posibilitar el contacto con iguales afectados (actúa limitando la despersonalización y la depositación desafectivizada de los estereotipos) y abordar creencias estereotipadas básicas como la responsabilidad, la impredecibilidad y la peligrosidad. Un resumen de los componentes críticos de las intervenciones puede verse en la Figura 1.

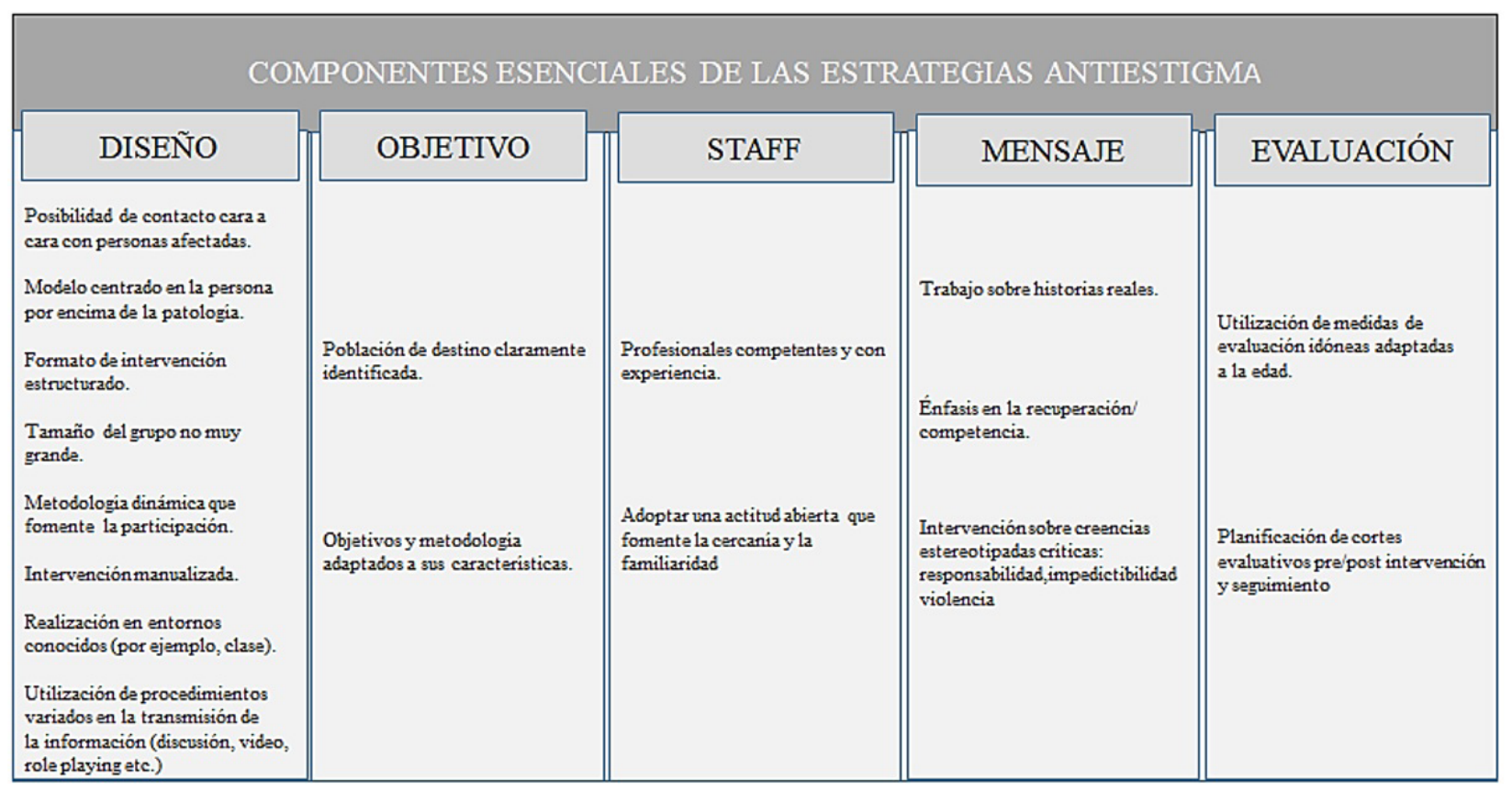

Figura 1. Componentes esenciales de las estrategias antiestigma

Presentamos en este trabajo los datos del pilotaje en nuestro contexto de un programa para la reducción del estigma en las aulas: el programa "Lo Hablamos". Se trata de una estrategia encuadrada dentro del II Plan Integral de Salud Mental de Andalucía 2008-2012 (2008) y que nace con el doble objetivo de sensibilizar a la población adolescente (de 14 a las 16 años) sobre 
la salud mental y promover un trato menos discriminatorio hacia las personas que padecen problemáticas en este ámbito. El programa ha sido diseñado por el Grupo de Sensibilización sobre Salud Mental de Andalucía, en el marco de la línea estratégica 1 decada4.

\section{Método}

\section{Participantes}

El estudio se llevó a cabo en Puerto Real, municipio de la provincia de Cádiz que cuenta actualmente con 41.650 habitantes, de los cuales un 21,68\% corresponde al grupo de edad menor de 20 años (Instituto de Estadística y Cartografía, 2018). Participaron en el mismo 315 alumnos de $3^{\circ}$ y $4^{\circ}$ de Educación Secundaria Obligatoria (ESO) de 5 de los 7 Institutos con los que actualmente cuenta la localidad. La distribución especifica por edades y sexo se integra en la Figura 2.

\begin{tabular}{|c|c|c|c|c|c|c|c|c|c|c|c|c|c|c|c|}
\hline & \multicolumn{2}{|c|}{$\mathrm{H}$} & \multicolumn{2}{|c|}{$\mathrm{M}$} & & \multicolumn{2}{|c|}{14} & \multicolumn{2}{|c|}{15} & \multicolumn{2}{|c|}{16} & \multicolumn{2}{|c|}{17} & \multicolumn{2}{|c|}{18} \\
\hline & $\mathrm{N}$ & $\%$ & $\mathrm{~N}$ & $\%$ & & $\mathrm{~N}$ & $\%$ & $\mathrm{~N}$ & $\%$ & $\mathrm{~N}$ & $\%$ & $\mathrm{~N}$ & $\%$ & $\mathrm{~N}$ & $\%$ \\
\hline SEXO & 170 & 54 & 145 & 46 & $\mathrm{EDAD}$ & 76 & 24.1 & 124 & 39.4 & 81 & 25.7 & 27 & 8.6 & 7 & 2.2 \\
\hline
\end{tabular}

Figura 2. Distribución de alumnos por edades y sexo

\section{Procedimiento}

El programa se implementó a través de una sesión de 3 horas de duración desarrollada dentro del horario escolar y en el propio contexto de la clase. En la puesta en marcha de la estrategia han participado profesionales de diferentes categorías (psicólogos clínicos, psiquiatras, enfermeros, terapeutas ocupacionales, trabajadores sociales) que se integraron de manera voluntaria en el proyecto. Todo el personal estaba adscrito a la Unidad de Gestión Clínica de Salud Mental del Hospital Universitario de Puerto Real. Las sesiones se llevaban a cabo por un equipo de 2 personas. Los profesores podían integrarse y participar en la actividad si lo consideraban oportuno.

Se diseñó una encuesta para la recogida de datos, que se entregaba al final de la sesión a cada participante. Su cumplimentación era voluntaria y las opiniones recogidas en ella completamente anónimas. Constaba de nueve ítems organizados en cuatro bloques: el primero, con datos básicos (edad, curso, centro educativo y sexo); el segundo, centrado en las impresiones generales sobre la actividad, compuesto por tres preguntas con opciones de respuesta evaluadas a través de una escala tipo Likert ( 0 a 5); un tercero que integraba cinco preguntas abiertas dirigidas hacia la valoración de aspectos formales de las actividades (validez aparente, estructura, compresión y dinámica de las tareas) y un cuarto encaminado a perfilar los aprendizajes personales, en este caso a través de un ítem en el que se solicitaba señalar los tres más significativos. La encuesta tenía una duración estimada de veinte minutos y se recoge en la Figura 3. 

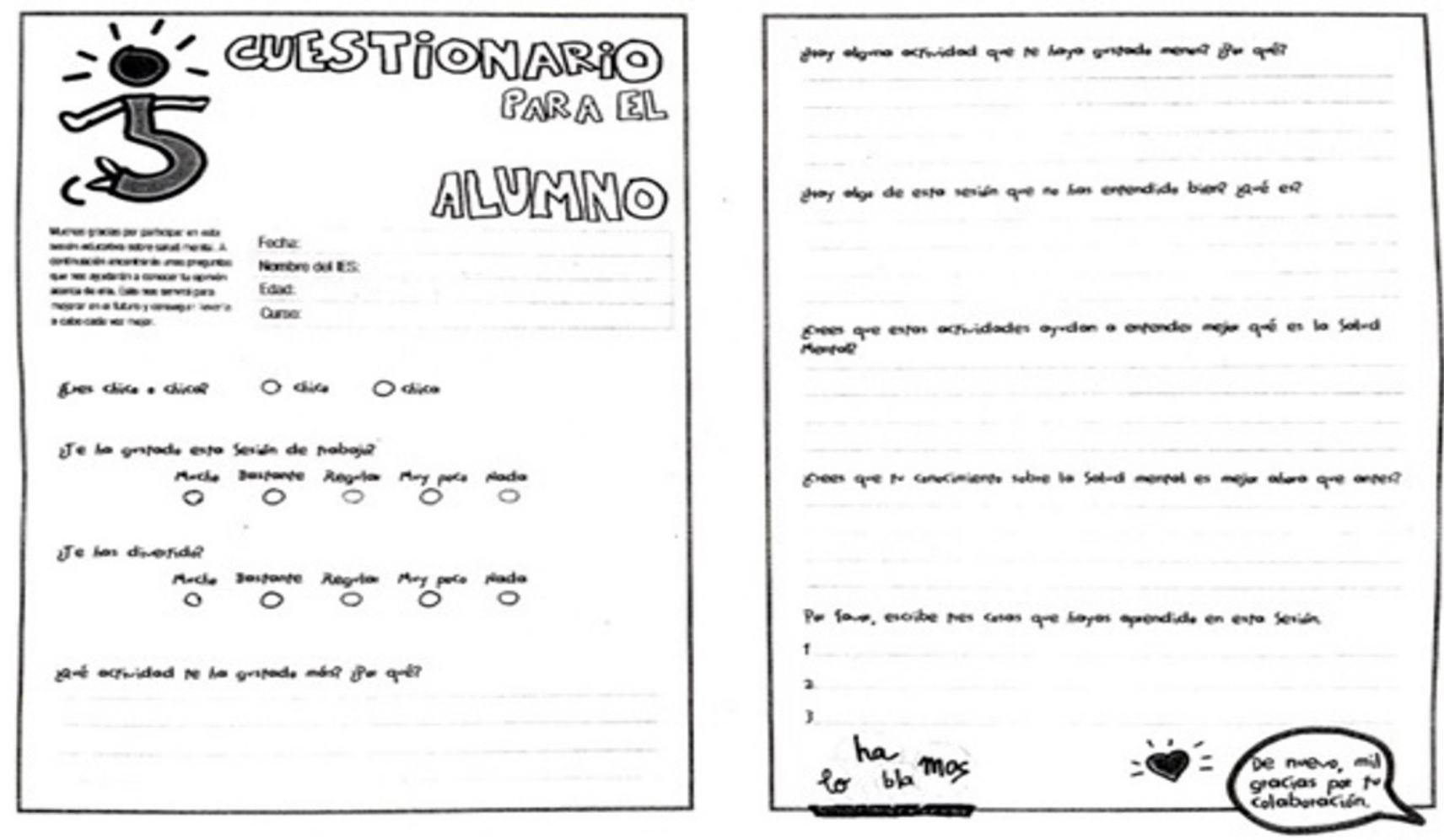

Figura 3. Cuestionario de valoración del programa

Padres, madres y tutores fueron informados de los objetivos del estudio y dieron su consentimiento para que sus hijos/as participaran en la investigación. En todo el proceso se garantizaron los estándares éticos de la declaración de Helsinki (World Medical Association, 2013) y sus enmiendas posteriores. Los datos se procesaron con el sotfware estadístico SPSS 24.

\section{Materiales}

El programa "Lo Hablamos" es una estrategia manualizada para la reducción del estigma en las aulas que cuenta con un importante grado de estructuración en sus contenidos que favorece la fidelización al procedimiento y su puesta en marcha por diferentes perfiles profesionales. Su objetivo básico es promover entre los participantes un conocimiento más adecuado sobre la salud mental a partir del cual se pueda incentivar un trato menos discriminatorio hacía las personas que padecen este tipo de problemas. Se pone el acento en la educación emocional y el cambio actitudinal, partiendo de la percepción propia de cada participante, estimulando la reflexión y la empatía hacía el otro.

Se articula alrededor de seis actividades grupales que inciden en las relaciones con el grupo de iguales (algunas están adaptadas del programa "Positive Mental Attitudes" con el permiso de Greater Glasgow and Clyde NHS). Son las siguientes:

Actividad n 1: Dinámica de Presentación: adjetivos (duración estimada: 10 minutos).

- Objetivos: toma de contacto con el grupo. Favorecer la confianza y el clima de trabajo. 
- Materiales: tarjetas para escribir adjetivos calificativos positivos.

Actividad n ${ }^{\circ}$ 2: Enfermedades que conoces (duración estimada: 15 minutos).

- Objetivos: conocer el concepto de salud mental a través del análisis grupal.

- Materiales: juegos de memoria y puesta en común.

Actividad no 3: ¿Qué opinas? (duración estimada: 20 minutos).

- Objetivos: afianzar una definición objetiva de la salud mental.

- Materiales: fichas con afirmaciones (V/F) para responder individualmente y en grupo.

Actividad no 4: Ponte en mi lugar (duración estimada: 30 minutos).

- Objetivos: profundizar en la percepción de la salud mental a través de la empatía.

- Materiales: ejercicio grupal de role playing con tarjetas (instrucciones) para la interacción.

Actividad no 5: La historia de James (duración estimada: 40 minutos).

- Objetivos: analizar la experiencia de una persona joven con problemas relacionados con la salud mental.

- Materiales: visionado del video "La historia de James" y trabajo grupal.

Actividad no 6: Debatir emociones e ideas erróneas (duración estimada: 40 minutos).

- Objetivos: reflexión general de los contenidos tratados en la sesión.

- Materiales: dinámica de grupo sobre preguntas guiadas.

\section{Resultados}

Las valoraciones realizadas sobre el programa son positivas y apuntan a que cuenta con una importante validez aparente e idoneidad en sus contenidos. El análisis de los datos se realizó siguiendo la estructura básica de la encuesta segmentando tres cluster: el primero centrado en la valoración de los aspectos formales del procedimiento (ítems 5-8); un segundo, que analiza la adecuación y dinámica de las tareas planteadas (ítems 1-4) y por último, un tercero, que se centra en su utilidad perciba por parte de los participantes (ítem 9).

En referencia a los aspectos de tipo formal (Figura 4), el primer dato a resaltar es que las actividades propuestas han alcanzado un elevado nivel de aceptación (83\%), solo una pequeña proporción de sujetos ha manifestado una evaluación negativa (3\%). El grado de comprensión expresada sobre las mismas es también muy alto $(92.7 \%)$, pocos alumnos las señalan como complejas, solo la $\mathrm{n}^{\mathrm{o}} 2(1.6 \%)$ y la $\mathrm{n}^{\mathrm{o}} 5(1.9 \%)$ exhiben un cierto nivel de relevancia en la valoración, sin ser esta mínimamente significativa. 


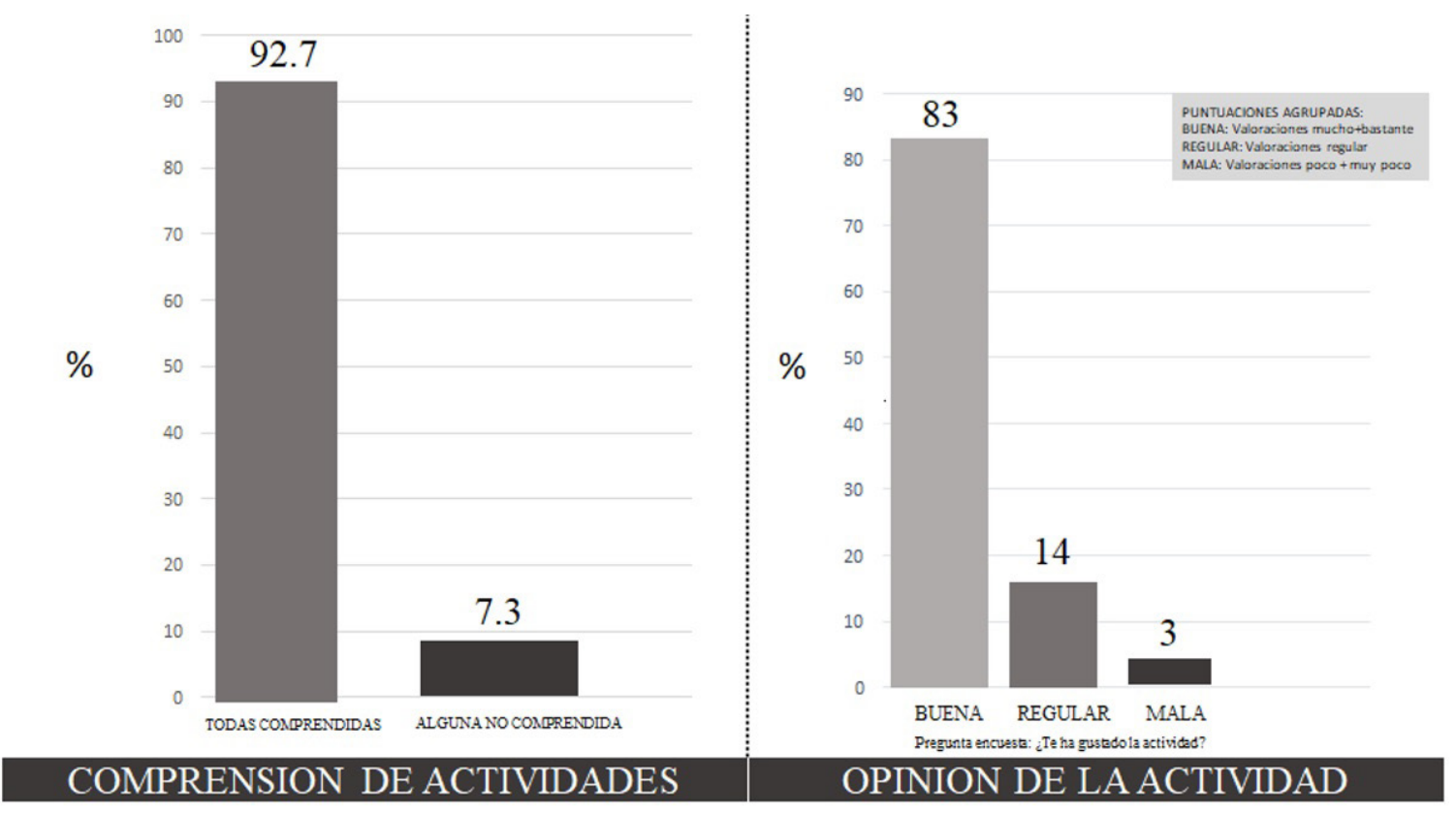

Figura 4. Aspectos formales de la intervención

La dinámica de trabajo que se genera con las tareas ha sido atractiva para los sujetos (Figura 5). Un número elevado de participantes $(72 \%)$ ha expresado que se ha divertido realizándolas, un menor porcentaje las ha considerado de manera más neutral $(23 \%)$ y solo una pequeña proporción las ha evaluado abiertamente como aburridas (5\%). Las actividad que ha generado un mayor grado de aceptación ha sido la $\mathrm{n}^{\mathrm{o}} 4$ ("Ponte en mi lugar") con un 55\% de las evaluaciones positivas, a mayor distancia se encuentran la n 3 (“¿Qué opinas?") con un 12\%, la n 6 ("Debatir emociones") con un 10\% y la n 5 ("La Historia de James") con un $9 \%$.

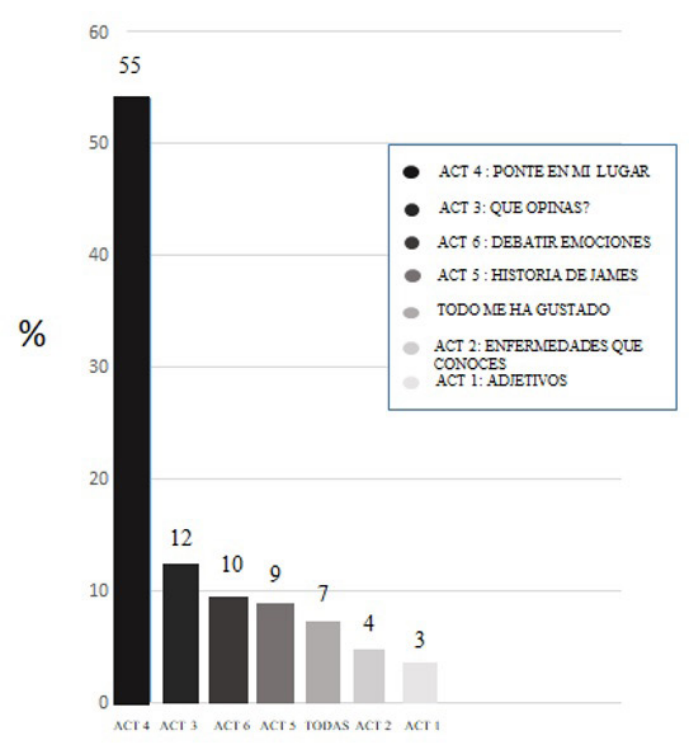

ACTIVIDADES MAS VALORADAS

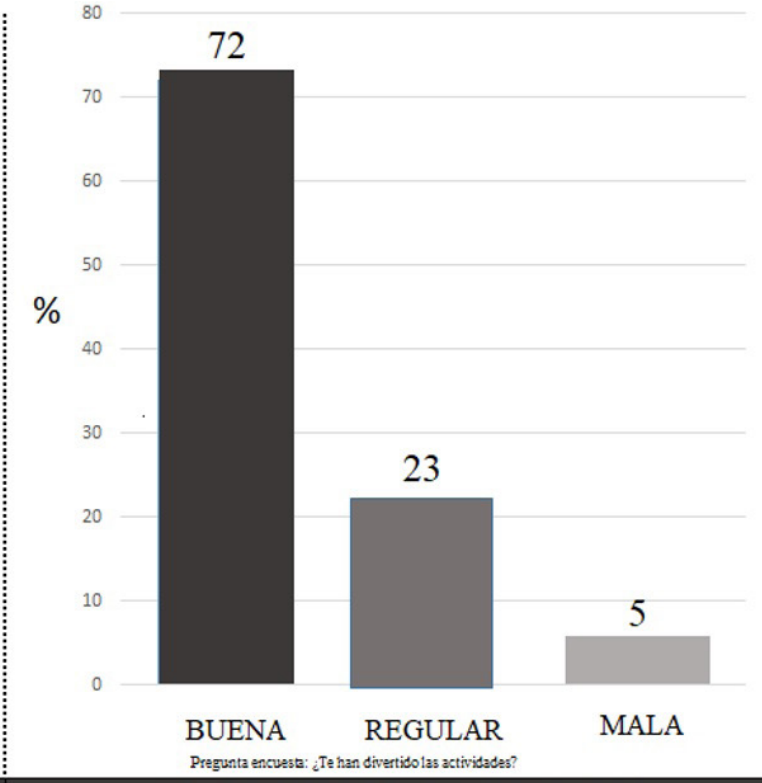

DINAMICA DE LAS ACTIVIDADES

Figura 5. Contenidos y dinámica de las actividades 
Los datos muestran que la valoración de las actividades está muy mediatizada por dos factores: el grado de dinamismo y la movilización afectiva que generan. En base a los mismos se perfila un claro gradiente en las preferencias asignadas a cada actividad, señalándose como mejor valoradas aquellas que en binomio racional-emocional enfatizan los aspectos afectivos y en el teórico-juego se decantan por este último como vehículo de los aprendizajes (Figura 6).

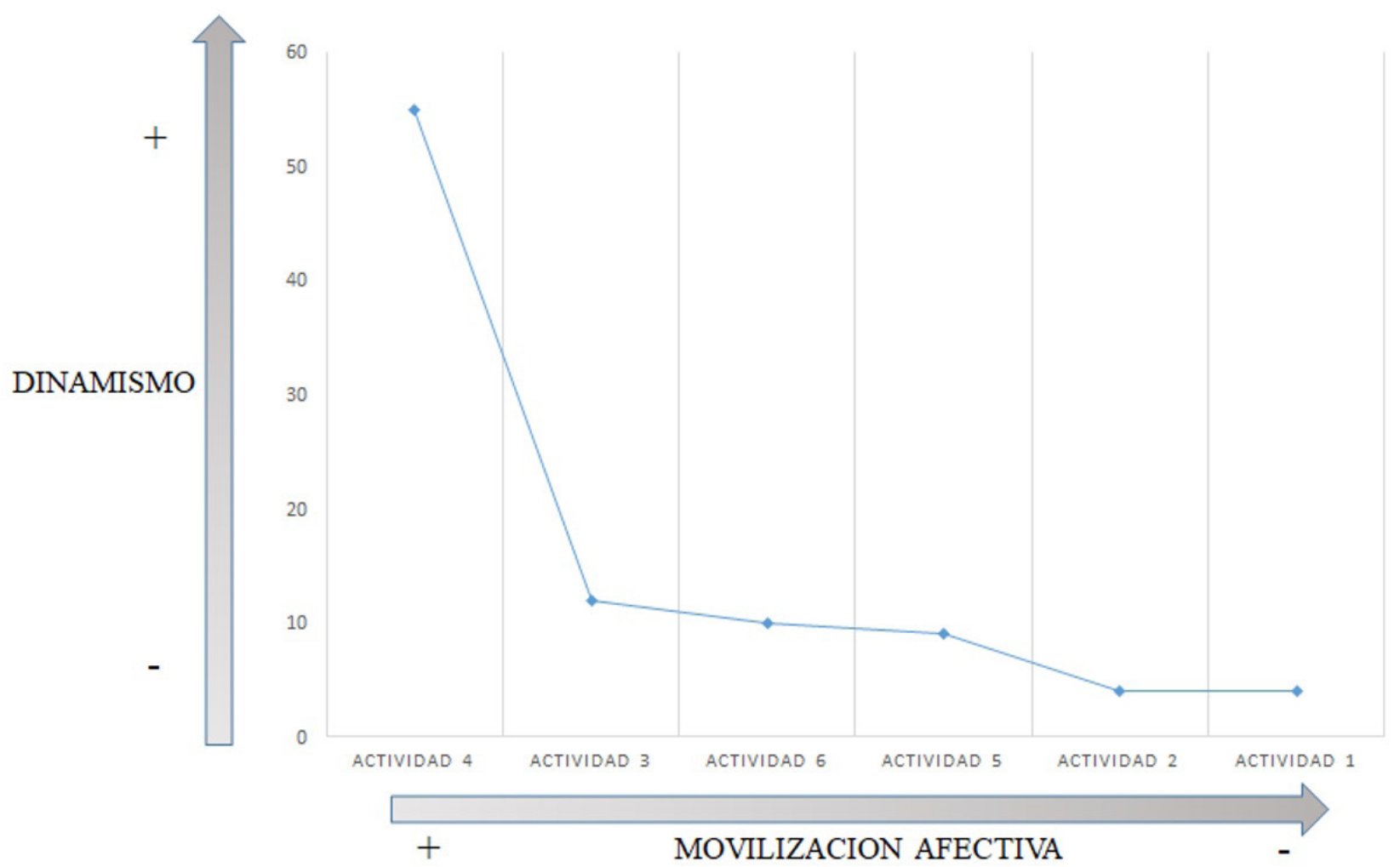

Figura 6. Gradiente en las preferencias por actividad

El programa ha logrado incrementar el nivel de conocimiento sobre los trastornos mentales. La práctica totalidad de los participantes (93\%) expresan que la actividad les ha ayudado a entender mejor a las personas que padecen este tipo de problemas y solo un porcentaje pequeño no han encontrado significativos los aprendizajes (7\%). Aparecen de forma clara cuatro adquisiciones que destacan sobre las demás y que son señaladas como relevantes: el saber más sobre la problemática mental (24.8\%), el comprender cómo se siente la persona afectada por este tipo de patologías (24.8\%), el poder normalizarlas (17.5\%) y el aprender a no discriminar (16.8\%). A destacar también que un porcentaje pequeño de los sujetos $(2.2 \%)$ expresa de manera abierta sentir o haber sentido el mismo tipo de emociones y de situaciones de discriminación que se señalan como potencialmente nocivas en la actividad (Figura 7). 


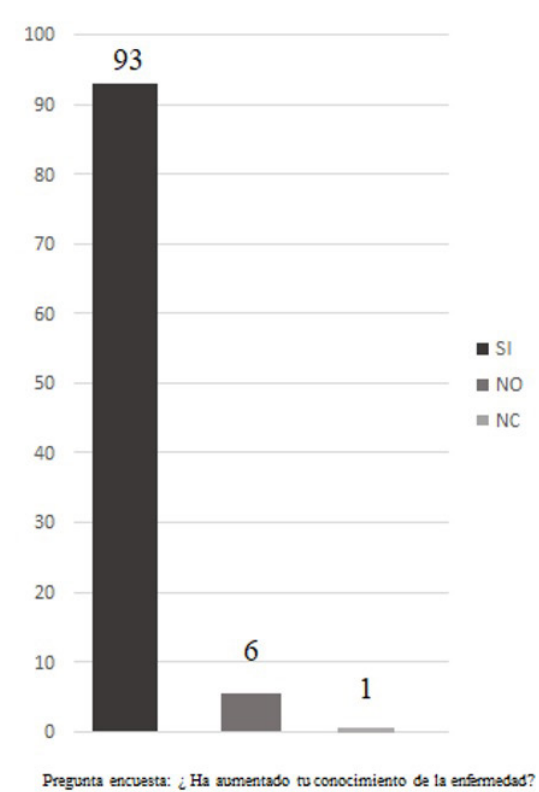

30

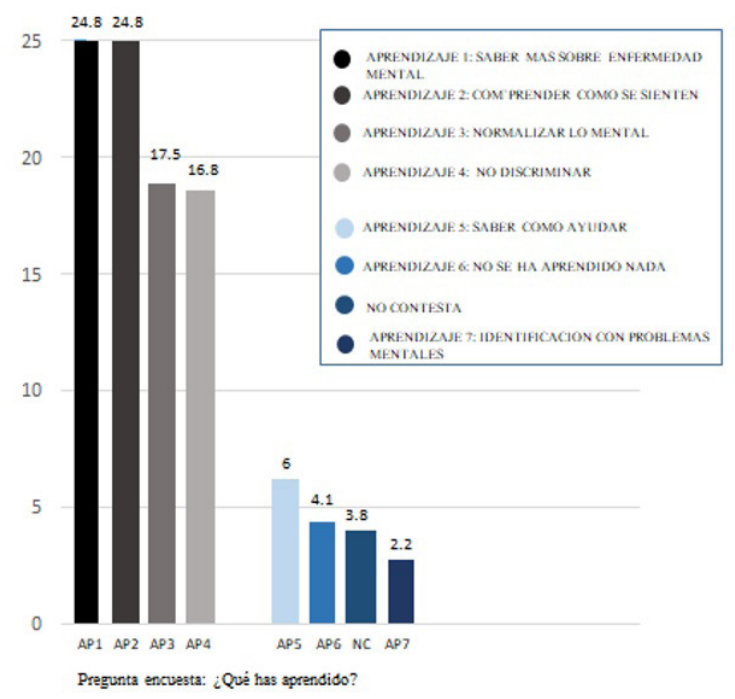

APRENDIZAJES MAS RELEVANTES

Figura 7. Utilidad percibida de la interveción

Es interesante hacer notar el papel que parece jugar la edad en la valoración de la utilidad percibida del programa. Los datos encontrados ponen de manifiesto que los juicios negativos se incrementan de manera notable a medida que la edad de los alumnos aumenta. El programa, aun siendo en general bien valorado, sí parece tener una población diana donde maximiza su validez aparente, que es el grupo de edad comprendido entre los 13-16 años. Por encima de estas edades los rangos de aceptabilidad son menores (Figura 8).

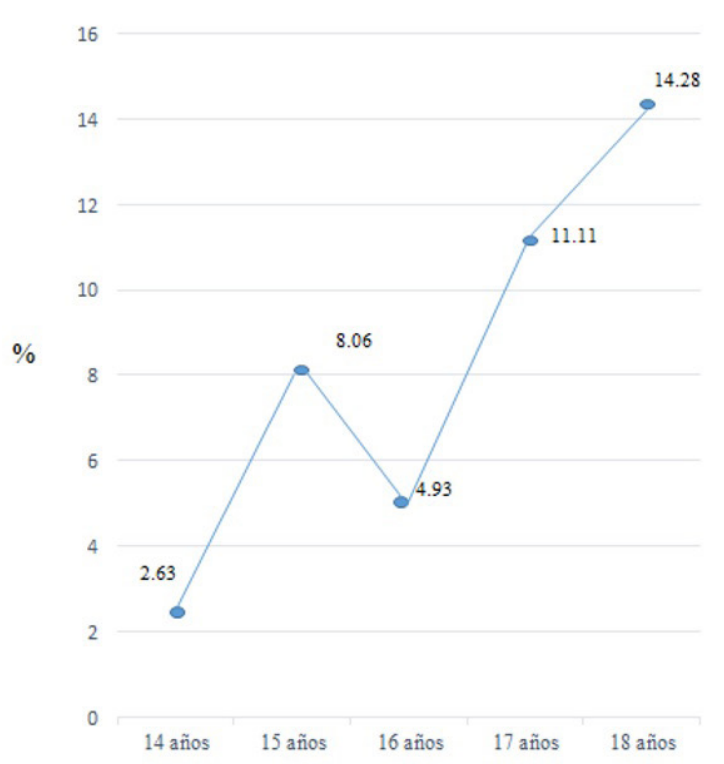

EVALUACIONES NEGATIVAS DE LA ACTIVIDAD

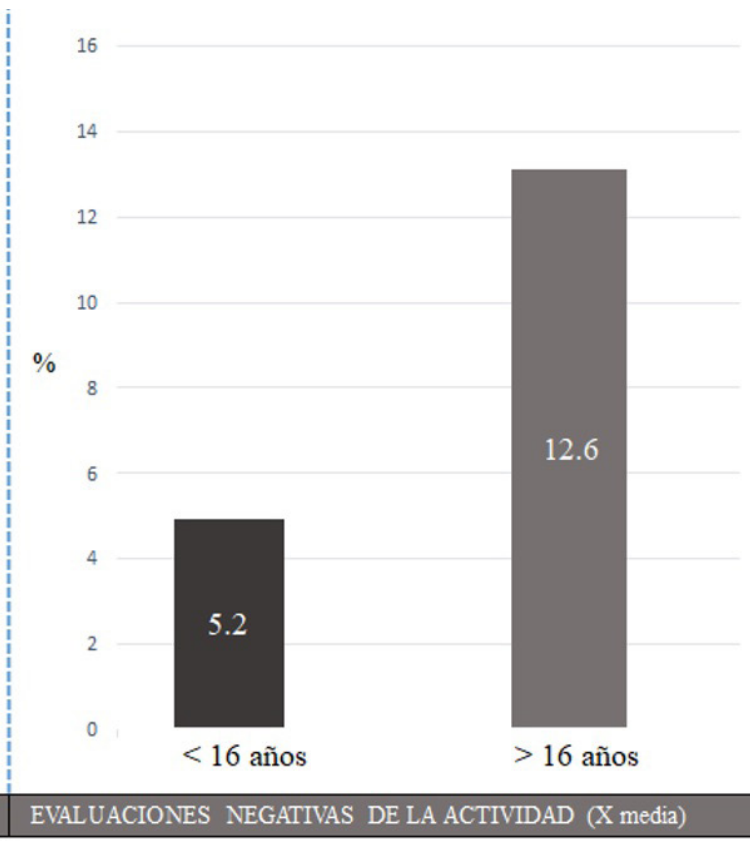

Figura 8. Evaluaciones negativas de la actividad 


\section{Discusión}

La adolescencia es un periodo especialmente interesante para desarrollar intervenciones antiestigma. Esta etapa representa en sí misma una oportunidad porque, por lo general, el joven se encuentra en una posición más creativa y flexible que el adulto, exhibe una mayor variabilidad en sus opiniones y estas son más sensibles al cambio (Bulanda et al., 2014; Kennedy et al., 2014; Lindow et al., 2020)

Uno de los primeros retos que se encuentran las intervenciones en esta franja de edad tiene que ver con los estilos de aprendizaje y preferencias propias de esta población. El diseño de las actividades ha de lograr un adecuado equilibrio entre la cualidad del mensaje que se quiere trasmitir (el qué) y el formato que se adopta para ello (el cómo) de manera que se optimicen al máximo la comprensión y los aprendizajes relevantes (Miller, Wakefield y Sani, 2017; Pittock, Meagher y Lawrie, 2019; Woolfson et al., 2009). En nuestro estudio el programa Lo Hablamos ha mostrado ser una estrategia atractiva, con contenidos fácilmente comprensibles y actividades dinámicas que han favorecido la implicación e interés del alumnado. Nuestros datos evidencian además que uno de los factores más relevantes para el éxito de una tarea es que sepa conjugar de manera adecuada la implicación personal y el juego como instrumento movilizador. Es relevante hacer notar en este sentido, que de todas las actividades integradas en el programa, la que ha obtenido mayor valoración ha sido aquella en la que se propone un aprendizaje más vivencial y empático con el juego de roles como herramienta ("ponte en mi lugar"). La estructura original del programa genera un nivel elevado de aceptación entre los alumnos de menor edad, disminuyendo la misma a medida que la edad avanza.

Al margen de que el programa resulte atractivo para el alumnado tiene que mostrarse eficaz y eficiente a la hora de conseguir los objetivos para los que ha sido diseñado. Como se ha señalado con anterioridad una de las variables más habitualmente relacionadas con la eficacia es que la estrategia permita el contacto cara a cara con personas afectadas. El programa Lo Hablamos no incluye el contacto directo, sino una presentación de video de 5 minutos de duración donde el protagonista expone con naturalidad sus temores iniciales cuando fue diagnosticado y que estrategias puso en marcha para poder superarlos. La utilización del contacto directo versus video está sujeta a controversia en la adolescencia, de hecho, parece que las recomendaciones que abogan por la interacción directa, habitualmente extraídas de estudios realizados con adultos, pueden no ser extrapolables a la población adolescente, sobre todo en sus edades más tempranas, en las que la utilización del video puede resultar igualmente eficaz, apuntándose además que la educación sin contacto puede ser mejor estrategia en estos rangos de edad (Chisholm et al., 2016; Janoušková et al., 2017; Koike et al., 2018). Parece ser que los efectos del contacto están claramente mediatizados por la edad, no es extraño que esto sea así, ya que buena parte de los adolescentes pueden no tener una idea diferenciada de la enfermedad, por lo que el contacto con personas afectadas y el intento de normalización puede confundir más que aclarar, produciendo un efecto paradójico, al incentivar el miedo a poder padecer una enfermedad mental y el incremento de las defensas de tipo fóbico y de distancia social.

Otro factor de relevancia tiene que ver con la secuencia y contenidos de las actuaciones. Hemos de recordar que la mayoría de los programas, incluido el nuestro, no solo buscan el reducir el estigma sino también el mejorar la identificación y el apoyo entre iguales. Esto último es de 
especial importancia si tenemos en cuenta que los amigos y personas del entorno cercano son la fuente primaria y más natural de apoyo en la adolescencia y constituyen el primer nivel de ayuda por encima de los propios profesionales (D'Avanzo et al., 2012; Ennis et al., 2019; Griggs, 2017; Hunt y Eisenberg, 2010; McGorry, Goldstone, Parker, Rickwood y Hickie, 2014). Trabajar desde un punto de vista exclusivamente educativo puede ser poco rentable o incluso contraproducente cuando hablamos de las enfermedades de mayor gravedad. En esta línea algunos trabajos han puesto de manifiesto que una mayor sensibilidad para el reconocimiento de enfermedades como la esquizofrenia suele inhibir la búsqueda de ayuda tanto en el círculo cercano como en el profesional (Yamasaki et al., 2016), además el deseo de apoyar por parte de los iguales se reduce de manera notable en estos casos (Attygalle, Perera y Jayamanne, 2017; Mason, Hart, Rossetto y Jorm, 2015; Svensson y Hansson, 2016; Yap, Reavley y Jorm, 2015). De ahí que se halla señalado la necesidad de actuar inicialmente y de manera extensiva sobre el estigma antes de instaurar programas educativos o de promoción de la salud, como medio para optimizar los rendimientos de estos últimos (Gardner, Filia, Killackey y Cotton, 2019; Gronholm, Henderson, Deb y Thornicroft, 2017; Thornicroft, 2016; Yamaguchi, Mino y Uddin, 2011). Podría resultar útil, en nuestra opinión, conceptualizar la intervención como un proceso continuo con al menos 3 fases: una primera encaminada a actuar específicamente sobre los prejuicios y el estigma; una segunda, centrada en la educación, que promoviera el conocimiento y la identificación precoz $\mathrm{y}$ una tercera que facilitara la adquisición de estrategias de afrontamiento y ayuda eficaz. Esta última cuestión es de especial transcendencia y aparece a menudo en un segundo plano, sin embargo pocos adolescentes van a estar preparados para dar una respuesta de apoyo eficaz si no se trabaja con ellos las habilidades instrumentales necesarias. Estrategias como el Mental Health First Aid se vienen moviendo en esta línea desde hace ya algunos años y recientemente han sido adaptadas para su uso en esta población (Hart, Mason, Kelly, Cvetkovski y Jorm, 2016; Hart et al., 2018; Ross et al., 2012). El programa Lo Hablamos representa, en nuestro caso, una primera línea de intervención sobre el estigma que se integra dentro de una estrategia global encaminada a diseñar un paquete de actuaciones que permitan la promoción de la salud en las aulas desde el concepto de competencia y la incentivación de valores como la promoción del respeto, la empatía y la aceptación de la diversidad.

Existe en la actualidad una importante variabilidad en cuanto a la extensión temporal de las intervenciones. El programa Lo Hablamos, en su formato original, está diseñado para una duración de 2,5 horas que, en nuestro caso, fueron ampliadas hasta 3 lo que podría considerarse una duración media. Se han comunicado resultados positivos con actuaciones de duración muy variada, incluyendo formatos muy cortos, pero también se ha señalado de manera consistente que, en la mayoría de los casos, las intervenciones pierden eficacia con el paso del tiempo y que incluso en algunas de ellas no se han producido cambios a nivel de prejuicios y actitudes discriminatorias (Corrigan, Michaels y Morris, 2015; Pinto-Foltz, Logsdon y Myers, 2011). Cada vez parece más claro que la mejora del conocimiento no se vincula de manera simple y automática a una reducción del estigma y que este último está arraigado en creencias más profundas pocas veces explicitadas de manera abierta. Aunque todavía no hay constancia empírica de cuál podría ser la duración más adecuada de las intervenciones sí parece lógico pensar que en los formatos más cortos la actuación sobre estas creencias será más recortada y que quizá por ello su eficacia decaiga con el tiempo por una reactivación de las mismas. La mejora inicial de las actitudes que se informa en 
algunos estudios puede tener poco poder predictivo sobre las conductas estigmatizantes o discriminatorias que podrían quedar inalteradas en un nivel más implícito. De hecho, algunos autores argumentan que la existencia de actitudes implícitas no adecuadamente medidas puede estar contaminando buena parte de los resultados encontrados en las investigaciones sobre el estigma en el contexto escolar (González-Sanguino, Muñoz, Castellanos, Pérez-Santos y Orihuela-Villameriel, 2019; O’Driscoll et al., 2012; O'Driscoll, Heary, Hennessy y McKeague, 2015a; Silke et al., 2017). Formatos muy cortos quizá logren cambios actitudinales explícitos, pero van a tener un efecto muy recortado sobre los estereotipos y prejuicios básicos hacia la enfermedad mental (Ke et al., 2015) por lo que, a nuestro juicio, es necesario optar por intervenciones más globales y extensivas para optimizar los rendimientos. En este sentido los datos que hemos encontrado en el pilotaje del procedimiento en nuestro contexto apuntan a que la extensión del mismo tendría que ser ampliada ya que el formato original no permite dedicar tiempo suficiente a los contenidos más relevantes y valorados por el alumnado que son los que se sitúan en la parte final del mismo. De igual forma creemos que es necesario realizar una reorganización de los ejercicios, si tenemos en cuenta el gradiente encontrado en las valoraciones de los alumnos las actividades 1 y 2 ("adjetivos" y "las enfermedades que conoces") parecen resultar menos atractivas y desempeñan además un papel secundario dentro del programa ya que su función aparente está en movilizar y generar una dinámica grupal óptima algo que, a nuestro juicio, podría lograrse de igual manera con otro tipo de tareas que exhibieran además una mayor vinculación con los contenidos críticos a trabajar dentro de la estrategia. Nuestro equipo de trabajo está actualmente diseñando nuevas tareas para incluir en el procedimiento.

La percepción del estigma se ha señalado de manera consistente como un factor inhibidor de la búsqueda de ayuda, de hecho, en buena parte de los casos, la explicitación de las dificultades emocionales suele estar asociada a un temor a ser reconocido por el resto de los compañeros (Mulfinger et al., 2019; Radez et al., 2020). En nuestros datos un $2.2 \%$ de los alumnos reconoce el haberse sentido identificado a nivel personal con los contenidos abordados. Este porcentaje, que en principio podría considerarse bajo, puede reflejar en realidad la punta del iceberg, en este caso con los casos más graves, y estar enmascarando una mayor proporción de problemáticas de corte, quizá menos grave, pero de evidente relevancia para la adaptación y el bienestar psicológico de los afectados, que permanecen silentes. El cómo acercarnos a este tipo de realidades larvadas continúa siendo hoy en día un reto. Recientemente se ha señalado el papel potencial que las nuevas tecnologías, y en particular del uso de internet, pueden jugar a la hora de educar y sensibilizar sobre temas de salud mental a la población adolescente, tanto para favorecer el contacto y la confianza con los profesionales, como el apoyo entre iguales, llegándose a desarrollar algunas apps con esta finalidad (Ali, Farrer, Gulliver y Griffiths, 2015; Álvarez-Jiménez, Gleeson, Rice, González-Blanch y Bendall, 2016; Corrigan, 2016; Naslund, Aschbrenner, Marsch y Bartels, 2016; Pretorius, Chambers y Coyle, 2019; Punukollu et al., 2020). Evidentemente estas nuevas tecnologías forman parte del cotidiano del adolescente, le resultan conocidas y atractivas y por tanto son metodologías con un enorme potencial. Diseñar estrategias que incluyan, junto con la actuación in situ, algún apoyo adicional con estos procedimientos puede, no solo facilitar el acceso a contenidos de ayuda para la población vulnerable, sino también servir de profundización y de búsqueda de estrategias de apoyo eficaces a los alumnos más sensibilizados. 
Parece claro que estamos ante un campo de actuación relevante y los resultados acumulados son alentadores, quedan, sin embargo aún, muchas cuestiones por dilucidar que aluden tanto a los aspectos formales de los procedimientos como a los contenidos que en ellos se deben incluir. El programa Lo Hablamos es una estrategia que, de inicio, parece atractiva, aunque es necesario someterla a estudios más rigurosos que evalúen su eficacia y, muy posiblemente, enriquecerla con modificaciones que posibiliten una mayor accesibilidad al alumnado y potencien la posibilidad de crear redes de apoyo entre iguales.

\section{Referencias}

Ali, K., Farrer, L., Gulliver, A., y Griffiths, K. M. (2015). Online peer-to-peer support for young people with mental health problems: a systematic review. JMIR mental health, 2(2), e19.

Álvarez-Jiménez, M., Gleeson, J. F., Rice, S., González-Blanch, C., y Bendall, S. (2016). Online peer-to-peer support in youth mental health: seizing the opportunity. Epidemiology and psychiatric sciences, 25(2), 123-126.

Andersen, B. J. (2013). The effects of preventive mental health programmes in secondary schools. En Papers from the 38th IHF World Hospital Congress in Oslo (Vol. 49, No. 4, p. 48).

Andrés-Rodríguez, L., Pérez-Aranda, A., Feliu-Soler, A., Rubio-Valera, M., Aznar-Lou, I., Serrano-Blanco, A., ... y Luciano, J. V. (2017). Effectiveness of the "what's up!" intervention to reduce stigma and psychometric properties of the Youth Program Questionnaire (YPQ): Results from a cluster non-randomized controlled trial conducted in Catalan high schools. Frontiers in psychology, 8, 1608.

Angermeyer, M. C., Carta, M. G., Matschinger, H., Millier, A., Refaï, T., Schomerus, G., y Toumi, M. (2016). Cultural differences in stigma surrounding schizophrenia: comparison between Central Europe and North Africa. The British Journal of Psychiatry, 208(4), 389-397.

Attygalle, U. R., Perera, H., y Jayamanne, B. D. W. (2017). Mental health literacy in adolescents: ability to recognise problems, helpful interventions and outcomes. Child and adolescent psychiatry and mental health, 11(1), 38.

Baba, Y., Nemoto, T., Tsujino, N., Yamaguchi, T., Katagiri, N., y Mizuno, M. (2017). Stigma toward psychosis and its formulation process: prejudice and discrimination against early stages of schizophrenia. Comprehensive psychiatry, 73, 181-186.

Barry, C. L., McGinty, E. E., Pescosolido, B. A., y Goldman, H. H. (2014). Stigma, discrimination, treatment effectiveness, and policy: public views about drug addiction and mental illness. Psychiatric Services, 65(10), 1269-1272.

Bentham, C., Daunt, A., Taylor, S., y Simmons, M. (2013). Mental health workshops delivered by medical students in Cambridge secondary schools: an evaluation of learning. Psychiatria Danubina, 25, S224-230.

Bulanda, J. J., Bruhn, C., Byro-Johnson, T., y Zentmyer, M. (2014). Addressing mental health stigma among young adolescents: evaluation of a youth-led approach. Health \& Social Work, 39(2), 73-80.

Cangas, A. J y Galván, B., (2020). Estudio preliminar de la utilidad de un programa de realidad virtual contra el estigma en salud mental. Paper publicado en XXVIII Congreso del INFAD de Psicología y Educación, Universidad Transilvania de Brasov, Rumanía. 
Cangas, A. J., Navarro, N., Aguilar-Parra, J. M., Trigueros, R., Gallego, J., Zárate, R., y Gregg, M. (2019). Analysis of the Usefulness of a Serious Game to Raise Awareness about Mental Health Problems in a Sample of High School and University Students: Relationship with Familiarity and Time Spent Playing Video Games. Journal of clinical medicine, 8(10), 1504. Cangas, A. J., Navarro, N., Parra, J., Ojeda, J. J., Cangas, D., Piedra, J. A., y Gallego, J. (2017). Stigma-Stop: A serious game against the stigma toward mental health in educational settings. Frontiers in psychology, 8, 1385.

Casañas, R., Arfuch, V. M., Castellví, P., Gil, J. J., Torres, M., Pujol, A., ... y Caussa, A. (2018). "EspaiJove. net"-a school-based intervention programme to promote mental health and eradicate stigma in the adolescent population: study protocol for a cluster randomised controlled trial. BMC public health, 18(1), 939.

Chandra, A., y Minkovitz, C. S. (2006). Stigma starts early: Gender differences in teen willingness to use mental health services. Journal of adolescent health, 38(6), 754-e1.

Chisholm, K., Patterson, P., Torgerson, C., Turner, E., Jenkinson, D., y Birchwood, M. (2016). Impact of contact on adolescents' mental health literacy and stigma: the SchoolSpace cluster randomised controlled trial. BMJ open, 6(2), e009435.

Choudhry, F. R., Mani, V., Ming, L. C., y Khan, T. M. (2016). Beliefs and perception about mental health issues: a meta-synthesis. Neuropsychiatric disease and treatment, 12, 2807.

Clement, S., Schauman, O., Graham, T., Maggioni, F., Evans-Lacko, S., Bezborodovs, N., ... y Thornicroft, G. (2015). What is the impact of mental health-related stigma on helpseeking? A systematic review of quantitative and qualitative studies. Psychological medicine, 45(1), 11-27.

Corrigan, P. W. (2016). Lessons learned from unintended consequences about erasing the stigma of mental illness. World Psychiatry, 15(1), 67-73.

Corrigan, P. W., Bink, A.B., Fokuo, J.K., y Schmidt, A. (2015). The public stigma of mental illness means a difference between you and me, Psychiatry Research, 226, 186-191.

Corrigan, P., y Lundin, R. (2012). Coming out proud to eliminate the stigma of mental illness manual. Chicago, IL: National Consortium on Stigma and Empowerment.

Corrigan, P., Michaels, P. J., y Morris, S. (2015). Do the effects of antistigma programs persist over time? Findings from a meta-analysis. Psychiatric Services, 66(5), 543-546.

Corrigan, P. W., Michaels, P. J., Powell, K., Bink, A., Sheehan, L., Schmidt, A., ... y Al-Khouja, M. (2016). Who comes out with their mental illness and how does it help?. The Journal of nervous and mental disease, 204(3), 163.

Corrigan, P. W., Powell, K. J., y Al-Khouja, M. A. (2015). Examining the impact of public service announcements on help seeking and stigma: Results of a randomized controlled trial. The Journal of nervous and mental disease, 203(11), 836-842.

Corrigan, J., Stephan, S. H., Hong, L. E., y Reeves, G. (2015). School-based approaches to reducing the duration of untreated psychosis. Child and Adolescent Psychiatric Clinics, 24(2), 335-351.

D’Avanzo, B., Barbato, A., Erzegovesi, S., Lampertico, L., Rapisarda, F., y Valsecchi, L. (2012). Formal and informal help-seeking for mental health problems. A survey of preferences of Italian students. Clinical practice and epidemiology in mental health: CP \& EMH, 8, 47. 
Deb, T., Lempp, H., Bakolis, I., Vince, T., Waugh, W., y Henderson, C. (2019). Responding to experienced and anticipated discrimination (READ): anti-stigma training for medical students towards patients with mental illness-study protocol for an international multisite non-randomised controlled study. BMC medical education, 19(1), 41.

Dolphin, L., y Hennessy, E. (2017). Labelling effects and adolescent responses to peers with depression: an experimental investigation. BMC psychiatry, 17(1), 228.

Ennis, E., McLafferty, M., Murray, E., Lapsley, C., Bjourson, T., Armour, C., ... y O’Neill, S. (2019). Readiness to change and barriers to treatment seeking in college students with a mental disorder. Journal of affective disorders, 252, 428-434.

Gardner, A., Filia, K., Killackey, E., y Cotton, S. (2019). The social inclusion of young people with serious mental illness: A narrative review of the literature and suggested future directions. Australian \& New Zealand Journal of Psychiatry, 53(1), 15-26.

Georgakakou-Koutsonikou, N., y Williams, J. M. (2017). Children and young people's conceptualizations of depression: a systematic review and narrative meta-synthesis. Child: care, health and development, 43(2), 161-181.

Giannakopoulos, G., Assimopoulos, H., Petanidou, D., Tzavara, C., Kolaitis, G., y Tsiantis, J. (2012). Effectiveness of a school-based intervention for enhancing adolescents' positive attitudes towards people with mental illness. Mental illness, 4(2).

González-Sanguino, C., Muñoz, M., Castellanos, M. A., Pérez-Santos, E., y Orihuela-Villameriel, T. (2019). Study of the relationship between implicit and explicit stigmas associated with mental illness. Psychiatry research, 272, 663-668.

González-Sanguino, C., Potts, L. C., Milenova, M., y Henderson, C. (2019). Time to Change's social marketing campaign for a new target population: results from 2017 to 2019 . BMC psychiatry, 19(1), 417.

Griffiths, K. M., Carron-Arthur, B., Parsons, A., y Reid, R. (2014). Effectiveness of programs for reducing the stigma associated with mental disorders. A meta-analysis of randomized controlled trials. World psychiatry, 13(2), 161-175.

Griggs, S. (2017). Hope and mental health in young adult college students: an integrative review. Journal of psychosocial nursing and mental health services, 55(2), 28-35.

Gronholm, P. C., Henderson, C., Deb, T., y Thornicroft, G. (2017). Interventions to reduce discrimination and stigma: the state of the art. Social psychiatry and psychiatric epidemiology, 52(3), 249-258.

Gronholm, P. C., Nye, E., y Michelson, D. (2018). Stigma related to targeted school-based mental health interventions: a systematic review of qualitative evidence. Journal of affective disorders, 240, 17-26.

Hanisch, S. E., Twomey, C. D., Szeto, A. C., Birner, U. W., Nowak, D., y Sabariego, C. (2016). The effectiveness of interventions targeting the stigma of mental illness at the workplace: a systematic review. BMC psychiatry, 16(1), 1.

Harris, J. I., Leskela, J., Lakhan, S., Usset, T., DeVries, M., Mittal, D., y Boyd, J. (2019). Developing Organizational Interventions to Address Stigma Among Mental Health Providers: A Pilot Study. Community mental health journal, 55(6), 924-931. 
Hart, L. M., Mason, R. J., Kelly, C. M., Cvetkovski, S., y Jorm, A. F. (2016). 'teen Mental Health First Aid': a description of the program and an initial evaluation. International journal of mental health systems, 10(1), 3 .

Hart, L. M., Morgan, A. J., Rossetto, A., Kelly, C. M., Mackinnon, A., y Jorm, A. F. (2018). Helping adolescents to better support their peers with a mental health problem: A clusterrandomised crossover trial of teen Mental Health First Aid. Australian \& New Zealand Journal of Psychiatry, 52(7), 638-651.

Hartog, K., Hubbard, C. D., Krouwer, A. F., Thornicroft, G., Kohrt, B. A., y Jordans, M. J. (2020). Stigma reduction interventions for children and adolescents in low-and middleincome countries: Systematic review of intervention strategies. Social Science \& Medicine, 112749 .

Hayes, D., Moore, A., Stapley, E., Humphrey, N., Mansfield, R., Santos, J., ... y Deighton, J. (2019). School-based intervention study examining approaches for well-being and mental health literacy of pupils in Year 9 in England: study protocol for a multischool, parallel group cluster randomised controlled trial (AWARE). BMJ open, 9(8), e029044.

Heim, E., Henderson, C., Kohrt, B. A., Koschorke, M., Milenova, M., y Thornicroft, G. (2020). Reducing mental health-related stigma among medical and nursing students in low-and middle-income countries: a systematic review. Epidemiology and psychiatric sciences, 29. Instituto de Estadística y Cartografía de Andalucía [IECA] (2018). Andalucía pueblo a pueblo - fichas municipales [Base de datos]. Recuperado de http://www.juntadeandalucia.es/ institutodeestadisticaycartografia/sima/ficha.htm?mun=11028

Hunt, J., y Eisenberg, D. (2010). Mental health problems and help-seeking behavior among college students. Journal of adolescent health, 46(1), 3-10.

Janoušková, M., Tušková, E., Weissová, A., Trančík, P., Pasz, J., Evans-Lacko, S., y Winkler, P. (2017). Can video interventions be used to effectively destigmatize mental illness among young people? A systematic review. European Psychiatry, 41, 1-9.

Junta de Andalucía, (2008). II Plan Integral de Salud Mental de Andalucía 2008-2012. Sevilla: Junta de Andalucía, Consejería de Salud.

Ke, S., Lai, J., Sun, T., Yang, M. M., Wang, J. C. C., y Austin, J. (2015). Healthy young minds: The effects of a 1-hour classroom workshop on mental illness stigma in high school students. Community mental health journal, 51(3), 329-337.

Kennedy, V., y Belgamwar, R. B. (2014). Impact of work experience placements on school students' attitude towards mental illness. The Psychiatric Bulletin, 38(4), 159-163.

Knaak, S., Modgill, G., y Patten, S. B. (2014). Key ingredients of anti-stigma programs for health care providers: a data synthesis of evaluative studies. The Canadian Journal of Psychiatry, 59(1_suppl), 19-26.

Koike, S., Yamaguchi, S., Ojio, Y., Ohta, K., Shimada, T., Watanabe, K., ... y Ando, S. (2018). A randomised controlled trial of repeated filmed social contact on reducing mental illnessrelated stigma in young adults. Epidemiology and psychiatric sciences, 27(2), 199-208.

Lansu, T. A. (2018). Burn! How implicit and explicit attitudes predict early adolescents'"hot sauce" aggression toward classroom peers. Journal of experimental child psychology, 167, 423-432. 
Lindow, J. C., Hughes, J. L., South, C., Minhajuddin, A., Gutierrez, L., Bannister, E., ... y Byerly, M. J. (2020). The Youth Aware of Mental Health Intervention: Impact on Help Seeking, Mental Health Knowledge, and Stigma in US Adolescents. Journal of Adolescent Health.

Mason, R. J., Hart, L. M., Rossetto, A., y Jorm, A. F. (2015). Quality and predictors of adolescents' first aid intentions and actions towards a peer with a mental health problem. Psychiatry Research, 228(1), 31-38.

McGorry, P. D., Goldstone, S. D., Parker, A. G., Rickwood, D. J., y Hickie, I. B. (2014). Cultures for mental health care of young people: an Australian blueprint for reform. The Lancet Psychiatry, 1(7), 559-568.

McKeague, L., Hennessy, E., O’Driscoll, C., y Heary, C. (2015). Retrospective accounts of selfstigma experienced by young people with attention-deficit/hyperactivity disorder (ADHD) or depression. Psychiatric Rehabilitation Journal, 38(2), 158.

Mehta, N., Clement, S., Marcus, E., Stona, A. C., Bezborodovs, N., Evans-Lacko, S., ... y Koschorke, M. (2015). Evidence for effective interventions to reduce mental health-related stigma and discrimination in the medium and long term: systematic review. The British Journal of Psychiatry, 207(5), 377-384.

Mellor, C. (2014). School-based interventions targeting stigma of mental illness: systematic review. The Psychiatric Bulletin, 38(4), 164-171.

Miller, K., Wakefield, J. R., y Sani, F. (2017). On the reciprocal effects between multiple group identifications and mental health: A longitudinal study of Scottish adolescents. British Journal of Clinical Psychology, 56(4), 357-371.

Mittal, D., Corrigan, P., Drummond, K. L., Porchia, S., y Sullivan, G. (2016). Provider opinions regarding the development of a stigma-reduction intervention tailored for providers. Health Education \& Behavior, 43(5), 577-583.

Morgan, A. J., Reavley, N. J., Ross, A., San Too, L., y Jorm, A. F. (2018). Interventions to reduce stigma towards people with severe mental illness: Systematic review and metaanalysis. Journal of psychiatric research, 103, 120-133.

Mulfinger, N., Rüsch, N., Bayha, P., Müller, S., Böge, I., Sakar, V., y Krumm, S. (2019). Secrecy versus disclosure of mental illness among adolescents: I. The perspective of adolescents with mental illness. Journal of mental health, 28(3), 296-303.

Naslund, J. A., Aschbrenner, K. A., Marsch, L. A., y Bartels, S. J. (2016). The future of mental health care: peer-to-peer support and social media. Epidemiology and psychiatric sciences, 25(2), 113-122.

O’Driscoll, C., Heary, C., Hennessy, E., y McKeague, L. (2012). Explicit and implicit stigma towards peers with mental health problems in childhood and adolescence. Journal of Child Psychology and Psychiatry, 53(10), 1054-1062.

O’Driscoll, C., Heary, C., Hennessy, E., y McKeague, L. (2015a). Adolescents' explanations for the exclusion of peers with mental health problems: An insight into stigma. Journal of Adolescent Research, 30(6), 710-728.

O'Driscoll, C., Heary, C., Hennessy, E., y McKeague, L. (2015b). Adolescents' beliefs about the fairness of exclusion of peers with mental health problems. Journal of adolescence, 42, 59-67. 
Ojio, Y., Yonehara, H., Taneichi, S., Yamasaki, S., Ando, S., Togo, F., ... y Sasaki, T. (2015). Effects of school-based mental health literacy education for secondary school students to be delivered by school teachers: A preliminary study. Psychiatry and clinical neurosciences, 69(9), 572-579.

Pescosolido, B. A., Manago, B., y Monahan, J. (2019). Evolving public views on the likelihood of violence from people with mental illness: stigma and its consequences. Health Affairs, 38(10), 1735-1743.

Pinfold, V., Thornicroft, G., Huxley, P., y Farmer, P. (2005). Active ingredients in anti-stigma programmes in mental health. International Review of Psychiatry, 17(2), 123-131.

Pinto-Foltz, M. D., Logsdon, M. C., y Myers, J. A. (2011). Feasibility, acceptability, and initial efficacy of a knowledge-contact program to reduce mental illness stigma and improve mental health literacy in adolescents. Social science \& medicine, 72(12).

Pittock, A., Meagher, L., y Lawrie, S. M. (2019). Evaluating PsychEd: a mental health and wellbeing educational programme for secondary schools. BJPsych open, 5(3).

Pretorius, C., Chambers, D., y Coyle, D. (2019). Young People's Online Help-Seeking and Mental Health Difficulties: Systematic Narrative Review. Journal of medical Internet research, 21(11), e13873.

Punukollu, M., Leighton, E. L., Brooks, A. F., Heron, S., Mitchell, F., Regener, P., ... y Sharpe, H. (2020). SafeSpot: an innovative app and mental health support package for Scottish schools-a qualitative analysis as part of a mixed methods study. Child and Adolescent Mental Health, 25(2), 110-116.

Puolakka, K., Haapasalo-Pesu, K. M., Konu, A., Åstedt-Kurki, P., y Paavilainen, E. (2014). Mental health promotion in a school community by using the results from the well-being profile: an action research project. Health promotion practice, 15(1), 44-54.

Radez, J., Reardon, T., Creswell, C., Lawrence, P. J., Evdoka-Burton, G., y Waite, P. (2020). Why do children and adolescents (not) seek and access professional help for their mental health problems? A systematic review of quantitative and qualitative studies. European Child \& Adolescent Psychiatry, 1-29.

Ross, A. M., Hart, L. M., Jorm, A. F., Kelly, C. M., y Kitchener, B. A. (2012). Development of key messages for adolescents on providing basic mental health first aid to peers: a Delphi consensus study. Early intervention in psychiatry, 6(3), 229-238.

Sakellari, E., Sourander, A., Kalokerinou-Anagnostopoulou, A., y Leino-Kilpi, H. (2014). The impact of an educational mental health intervention on adolescents' perceptions of mental illness. Journal of psychiatric and mental health nursing, 21(7), 635-641.

Salerno, J. P. (2016). Effectiveness of universal school-based mental health awareness programs among youth in the United States: a systematic review. Journal of school health, 86(12), 922-931.

Sampogna, G., Bakolis, I., Robinson, E., Corker, E., Pinfold, V., Thornicroft, G., y Henderson, C. (2017). Experience of the Time to Change programme in England as predictor of mental health service users' stigma coping strategies. Epidemiology and psychiatric sciences, 26(5), 517-525.

Schachter, H. M., Girardi, A., Ly, M., Lacroix, D., Lumb, A. B., van Berkom, J., y Gill, R. (2008). Effects of school-based interventions on mental health stigmatization: a systematic review. Child and Adolescent Psychiatry and Mental Health, 2(1), 18. 
Schlier, B., y Lincoln, T. M. (2019). The stigma of mental illness: Testing for the implicit bias in diagnostic labels. Psychiatry research, 275, 221-227.

Setti, V. P. C., Loch, A. A., Modelli, A., de Almeida Rocca, C. C., Hungerbuehler, I., van de Bilt, M. T., ... y Rössler, W. (2019). Disclosing the diagnosis of schizophrenia: A pilot study of the 'Coming Out Proud'intervention. International Journal of Social Psychiatry, 65(3), 244-251.

Silke, C., Swords, L., y Heary, C. (2016). The development of an empirical model of mental health stigma in adolescents. Psychiatry research, 242, 262-270.

Silke, C., Swords, L., y Heary, C. (2017). The predictive effect of empathy and social norms on adolescents' implicit and explicit stigma responses. Psychiatry research, 257, 118-125.

Skre, I., Friborg, O., Breivik, C., Johnsen, L. I., Arnesen, Y., y Wang, C. E. A. (2013). A school intervention for mental health literacy in adolescents: effects of a non-randomized cluster controlled trial. BMC Public Health, 13(1), 873.

Stuart, H., Chen, S. P., Christie, R., Dobson, K., Kirsh, B., Knaak, S., ... y Modgill, G. (2014). Opening minds in Canada: targeting change. The Canadian Journal of Psychiatry, 59(1_ suppl), 13-18.

Sugumar, D., Fleming, O., y Ogden, K. (2019). A mental health programme for medical students. The clinical teacher, 16(4), 352-355.

Svensson, B., y Hansson, L. (2016). How mental health literacy and experience of mental illness relate to stigmatizing attitudes and social distance towards people with depression or psychosis: A cross-sectional study. Nordic journal of psychiatry, 70(4), 309-313.

Swords, L., Heary, C., y Hennessy, E. (2011). Factors associated with acceptance of peers with mental health problems in childhood and adolescence. Journal of Child Psychology and Psychiatry, 52(9), 933-941.

Szeto, A., Dobson, K. S., Luong, D., Krupa, T., y Kirsh, B. (2019). Workplace antistigma programs at the Mental Health Commission of Canada: Part 1. Processes and projects. The Canadian Journal of Psychiatry, 64(1_suppl), 5S-12S.

Thornicroft, G., Bakolis, I., Evans-Lacko, S., Gronholm, P. C., Henderson, C., Kohrt, B. A., ... y Sartorius, N. (2019). Key lessons learned from the indigo global network on mental health related stigma and discrimination. World Psychiatry, 18(2), 229-230.

Thornicroft, G., Mehta, N., Clement, S., Evans-Lacko, S., Doherty, M., Rose, D., ... y Henderson, C. (2016). Evidence for effective interventions to reduce mental-health-related stigma and discrimination. The Lancet, 387(10023), 1123-1132.

Wahl, O. E. (2002). Children's views of mental illness: a review of the literature. Psychiatric Rehabilitation Skills, 6(2), 134-158.

Walsh, D., y Foster, J. (2020). A Contagious Other? Exploring the Public's Appraisals of Contact with 'Mental Illness'. International Journal of Environmental Research and Public Health, 17(6), 2005.

Weare, K., y Markham, W. (2005). What do we know about promoting mental health through schools?. Promotion \& education, 12(3-4), 118-122.

Woolfson, R., Woolfson, L., Mooney, L., y Bryce, D. (2009). Young people's views of mental health education in secondary schools: a Scottish study. Child: care, health and development, 35(6), 790-798. 
World Medical Association. (2013). Declaration of Helsinki: Ethical principles for medical research involving human subjects. JAMA. 310(20), 2191-4. https://doi.org/10.1001/ jama.2013.281053.

Yamaguchi, S., Mino, Y., y Uddin, S. (2011). Strategies and future attempts to reduce stigmatization and increase awareness of mental health problems among young people: a narrative review of educational interventions. Psychiatry and clinical neurosciences, 65(5), 405-415.

Yamasaki, S., Ando, S., Shimodera, S., Endo, K., Okazaki, Y., Asukai, N., ... y Sasaki, T. (2016). The Recognition of Mental Illness, Schizophrenia Identification, and Help-Seeking from Friends in Late Adolescence. PloS one, 11(3).

Yang, J., Cervera, R. L., Tye, S. J., Ekker, S. C., y Pierret, C. (2018). Adolescent mental health education InSciEd Out: a case study of an alternative middle school population. Journal of translational medicine, 16(1), 84.

Yanos, P. T., Lucksted, A., Drapalski, A. L., Roe, D., y Lysaker, P. (2015). Interventions targeting mental health self-stigma: A review and comparison. Psychiatric Rehabilitation Journal, 38(2), 171-178

Yap, M. B. H., Reavley, N. J., y Jorm, A. F. (2015). Is the use of accurate psychiatric labels associated with intentions and beliefs about responses to mental illness in a friend? Findings from two national surveys of Australian youth. Epidemiology and psychiatric sciences, 24(1), 54-68. 